\title{
Diagnostic Value of Prenatal MR Imaging in the Detection of Brain Malformations in Fetuses before the 26th Week of Gestational Age
}

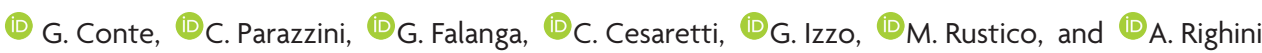

\begin{abstract}
BACKGROUND AND PURPOSE: In several countries, laws and regulations allow abortion for medical reasons within 24-25 weeks of gestational age. We investigated the diagnostic value of prenatal MR imaging for brain malformations within 25 weeks of gestational age.

MATERIALS AND METHODS: We retrospectively included fetuses within 25 weeks of gestational age who had undergone both prenatal and postnatal MR imaging of the brain between 2002 and 2014. Two senior pediatric neuroradiologists evaluated prenatal MR imaging examinations blinded to postnatal MR imaging findings. With postnatal MR imaging used as the reference standard, we calculated the sensitivity, specificity, positive predictive value, and negative predictive value of the prenatal MR imaging in detecting brain malformations.

RESULTS: One-hundred nine fetuses (median gestational age at prenatal MR imaging: 22 weeks; range, 21-25 weeks) were included in this study. According to the reference standard, 111 malformations were detected. Prenatal MR imaging failed to detect correctly 11 of the 111 malformations: 3 midline malformations, 5 disorders of cortical development, 2 posterior fossa anomalies, and 1 vascular malformation. Prenatal MR imaging misdiagnosed 3 findings as pathologic in the posterior fossa.
\end{abstract}

CONCLUSIONS: The diagnostic value of prenatal MR imaging between 21 and 25 weeks' gestational age is very high, with limitations of sensitivity regarding the detection of disorders of cortical development.

ABBREVIATIONS: GA = gestational age; US = ultrasonography

$\mathbf{P}$ renatal MR imaging of the brain is a technique increasingly used in clinical practice; it is generally performed as a secondlook investigation in case of abnormal or suspicious findings at prenatal ultrasonography (US). ${ }^{1}$

Prenatal MR imaging is often advocated as an important tool in parental counseling and decision-making regarding the fate of the pregnancy. ${ }^{2}$ In several countries, crucial decisions on pregnancy must be made before the 24th to 25th week of gestation because local laws and regulations allow abortion for medical reasons within this deadline. In these cases, a correct diagnosis should be reached early during pregnancy because performing

Received July 3, 2015; accepted after revision November 5

From the Departments of Radiology and Neuroradiology (G.C., C.P., C.C., G.I., A.R.) and Gynecology and Obstetrics (M.R.), Children's Hospital Vittore Buzzi, Milan, Italy; Department of Health Sciences (G.C.), University of Milan, Milan, Italy; and Department of Biopathology and Medical and Forensic Biotechnologies (G.F.), Section of Radiological Sciences, University of Palermo, Palermo, Italy.

Please address correspondence to Giorgio Conte, MD, Radiology and Neuroradiology Department, Children's Hospital Vittore Buzzi, Via Castelvetro 32, Milan, 20154, Italy; e-mail: giorgioconte.unimed@gmail.com

$\equiv$ Indicates article with supplemental on-line table.

Indicates article with supplemental on-line photos.

http://dx.doi.org/10.3174/ajnr.A4639 additional MR imaging follow-up is not compatible with legal time constraints. Moreover, an early correct diagnosis may have an important impact on the psychological well-being of the mother and may help the clinician in planning other diagnostic or therapeutic procedures.

To determine prenatal MR imaging accuracy, several studies have already compared its results with ones from postmortem examinations, ${ }^{3-5}$ postnatal MR imaging, ${ }^{6-11}$ or both postmortem examination and postnatal MR imaging. ${ }^{12,13}$ However, these studies were performed in small cohorts of fetuses, and they were focused on a single specific class of anomalies or accounted for few fetuses younger than 24-25 weeks' gestational age (GA), thus providing little information about the diagnostic accuracy of prenatal MR imaging performed at an early GA.

The purpose of our study was to assess the diagnostic value of prenatal MR imaging in the diagnosis of brain malformations, in a large cohort of fetuses (109 cases) within 25 weeks of GA, by using postnatal MR imaging as the reference standard.

\section{MATERIALS AND METHODS \\ Subjects}

We retrospectively included in our study all fetuses that underwent prenatal MR imaging at the Children's Hospital Vittore 
Buzzi from 2002 to 2014 and fulfilled the following criteria: 1) GA within 25 weeks at prenatal MR imaging examination; 2) neither termination of pregnancy, fetal death, nor stillbirth; and 3) at least 1 postnatal MR imaging examination of the brain performed at the Children's Hospital Vittore Buzzi or in other institutions and available for review. Mothers signed the specific informed consent for prenatal MR imaging in use in our institution, which also includes a disclaimer about data collection regarding clinical and imaging follow-up. The study complied with regulations in use in our institution for clinical review studies.

For each fetus, the US and/or clinical indications for the prenatal MR imaging, GA at prenatal MR imaging examination, and the child's age at postnatal MR imaging examination were recorded. Because our cases were referred from several different prenatal sonography centers, US was performed by operators with different experience levels. The US findings were collected from the examination reports. GA at prenatal MR imaging examination was determined by the mother's last menstrual period and by findings at first trimester US.

\section{Imaging Methods and Analysis}

All prenatal MR imaging examinations were performed on a $1.5 \mathrm{~T}$ system with a phased array abdominal or cardiac coil. Prenatal MR imaging protocol was standard clinical and state-of-the-art: It included T2-weighted single-shot FSE multiplanar sections (3- to 4-mm-thick sections; gap, $0.1 \mathrm{~mm}$; TR/TE, 3000/180 ms; in-plane resolution, $1.1 \mathrm{~mm}^{2}$ ); balanced steady-state multiplanar sections (contiguous 2- to 3-mm-thick sections); T1-weighted FSE multiplanar sections (5.5-mm-thick sections; TR/TE, 300/14 ms; turbo factor, 3; in-plane resolution, $1.4 \mathrm{~mm}^{2}$ ); and, in some cases, DWI sections (5.5-mm-thick sections; TR/TE, 1000/90 ms; b factor, $0-600 \mathrm{~s} / \mathrm{mm}^{2}$; FOV, $320 \times 320 \mathrm{~mm}$; matrix, $128 \times 128$ ).

Postnatal MR imaging sequences varied because they were performed at different institutions; however, all included sagittal T1-weighted images and T2-weighted images in at least 2 different anatomic planes with section thicknesses ranging from 3 to $5 \mathrm{~mm}$. When $>1$ postnatal MR imaging examination was performed, the first postnatal one was used as the reference for this study.

Two senior pediatric neuroradiologists (A.R., C.P.) with 12 years of experience in fetal MR imaging (approximately 150 fetal cases assessed yearly) evaluated prenatal MR imaging examinations separately; in cases of disagreement, they reached consensus in a second reading. At the time of the review of prenatal MR imaging examinations for this study, both readers were blinded to the patient's name and postnatal MR imaging findings; however, they were aware of the US and/or clinical indications for prenatal MR imaging and the GA at prenatal MR imaging. The 2 readers evaluated the postnatal MR imaging examinations in consensus with knowledge of the prenatal MR imaging findings.

For each fetus, the readers assessed the images for the following categories of brain malformations: 1) midline malformations, 2) disorders of cortical development, 3) posterior fossa anomalies, 4) vascular malformations, and 5) ventricular/subarachnoid space anomalies.
Table 1: Ultrasonographic and clinical indications for prenatal MR imaging

\begin{tabular}{lc}
\hline \multicolumn{1}{c}{ Indications for MR Imaging } & No. \\
\hline Ventricular dilation at US & 34 \\
Midline malformation at US & 20 \\
Posterior fossa anomalies at US & 17 \\
CMV infection & 14 \\
TTTS & 14 \\
Cysts at US & 2 \\
Vascular malformation at US & 2 \\
Brain anomalies in previous pregnancy & 2 \\
Cortical malformations at US & 1 \\
Toxoplasma infection & 1 \\
Polymalformation at US & 1 \\
Brain edema at US & 1 \\
\hline
\end{tabular}

Note:-TTTS indicates twin-to-twin transfusion syndrome; CMV, cytomegalovirus.

\section{Statistical Analysis}

With postnatal MR imaging used as the reference standard, we calculated the sensitivity, specificity, positive predictive value, and negative predictive value for each category of malformations on the basis of the final reading in consensus with the prenatal MR imaging. The agreement between the 2 readers in evaluating prenatal MR imaging was assessed by using the Cohen $\kappa$ statistic. We reported discrepancies between the US and MR imaging findings without providing statistics because US was performed by several centers, thus preventing us from reliably calculating the US performance in the detection of brain malformations.

\section{RESULTS}

Our study included 109 fetuses. The median GA at prenatal MR imaging examination was 22 weeks (range, 21-25 weeks). The median age at first postnatal MR imaging examination was 1 month (range, 0-60 months), corrected for delivery age $<37$ weeks. In 15 cases, a second postnatal MR imaging examination was performed at a median age of 8 months (range, 5-21 months).

US and clinical indications for prenatal MR imaging are summarized in Table 1.

According to the reference standard, 111 brain malformations were detected in 75 fetuses (median GA, 22 weeks; range, 19-25 weeks), while no brain malformation was detected in the remaining 34 fetuses (median GA, 22 weeks; range, 19-25 weeks). The brain malformations were categorized as follows: 27 midline malformations, 14 disorders of cortical development, 19 posterior fossa anomalies, 48 ventricular/subarachnoid space anomalies, and 3 vascular malformations (see Table 2 for details).

The prenatal MR imaging failed to detect correctly 11 of the 111 malformations: 3 midline malformations including 1 agenesis of septum pellucidum, 1 fused thalami (On-line Fig $1 E$ ), and 1 callosal hypoplasia; 5 disorders of cortical development including 3 focal polymicrogyrias and 2 periventricular nodular heterotopias (On-line Fig 2); 2 posterior fossa anomalies including 1 molar tooth malformation (On-line Fig $1 A-C$ ) and 1 Chiari type I malformation; and 1 vascular malformation consisting of a persistent falcine sinus. Prenatal MR imaging misdiagnosed 3 findings as pathologic (false-positives) in the posterior fossa: All were moderate $\left(<25^{\circ}\right)$ vermian cranial counterclockwise rotation angles 


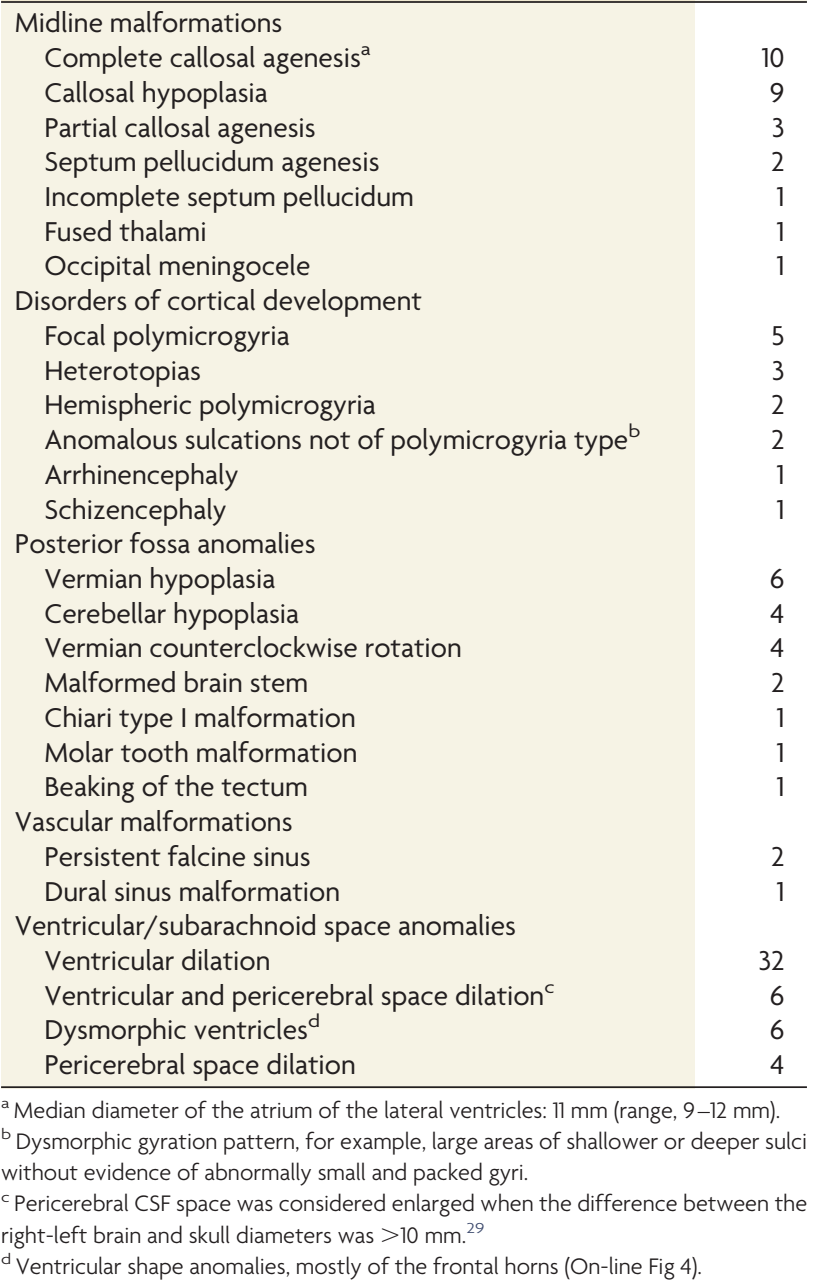

with respect to the fourth ventricle floor from a midsagittal section (On-line Fig 3).

Performance of the prenatal MR imaging according to the categories of brain malformations is summarized in the On-line Table.

The interobserver agreement in evaluating prenatal MR imaging was optimum, with $\kappa=0.99$ (95\% CI, $0.98-1.0)$, with reader disagreement in a case of arrhinencephaly.

Sonography failed to detect 28 of 111 malformations: 10 midline malformations ( 7 partial callosal agenesis, 1 septum pellucidum agenesis, 1 fused thalami, 1 complete callosal agenesis), 13 disorders of cortical development ( 7 polymicrogyrias, 3 periventricular nodular heterotopias, 2 anomalous sulcations not of polymicrogyria type, 1 arrhinencephaly), 4 posterior fossa anomalies (1 Chiari type I malformation, 1 molar tooth malformation, 1 beaking of the tectum, and 1 malformed brain stem), and 1 vascular malformation (persistent falcine sinus). Prenatal MR imaging detected 17 malformations more than sonography: 7 midline malformations ( 7 partial callosal agenesis), 8 disorders of cortical development (4 polymicrogyrias, 1 periventricular nodular heterotopia, 2 anomalous sulcations not of polymicrogyria type, and 1 arrhinencephaly), and 2 posterior fossa anomalies ( 1 beaking of the tectum and 1 malformed brain stem). Sonography did not add any findings to prenatal MR imaging.

\section{DISCUSSION}

This study demonstrates that in the context of a large referral fetal imaging center, with the assessment of pediatric neuroradiologists with $>10$ years' experience in fetal neuroimaging, the diagnostic value of prenatal MR imaging within 25 weeks of GA was very high, with important limitations of sensitivity regarding early detection of disorders of cortical development and, to a lesser extent, posterior fossa anomalies. Our data complement previous studies that investigated the performance of prenatal MR imaging in large cohorts of fetuses, but with mean GAs well above 25 weeks. ${ }^{6,7,10,11}$ Our study also showed an optimum interobserver agreement in evaluating prenatal MR imaging; however, this result may be because the 2 readers have worked together in the same hospital for $>10$ years. The readers were in disagreement in a case of arrhinencephaly studied at 23 weeks' GA. However, prenatal MR imaging can better depict olfactory sulci from 30 weeks' GA onward and olfactory bulbs from 30 to 34 weeks' GA. ${ }^{14}$

The median GA at prenatal MR imaging of our population reflects our national legislation that does not allow termination of pregnancy for medical reasons after the 23rd week of gestation. This highlighted the unmet need to investigate the effectiveness of prenatal MR imaging in detecting fetal malformations at an earlier gestational age because the counseling at this age range is a daily task of fetal medicine centers in our and other countries.

The range of children's ages at postnatal MR imaging was quite large, from 0 to 60 months, with a median value of 1 month. We can hypothesize that these data reflect the indications for postnatal MR imaging examinations being performed early because of abnormalities detected prenatally and others being performed later for an abnormal child development, such as seizure, neurodevelopment delay, and so forth. Fifteen children underwent a second postnatal MR imaging as a follow-up for the ventricular and/or pericerebral space enlargement. These second MR imaging examinations did not provide any findings in addition to the first MR imaging examinations and thus did not influence our analysis.

The main clinical and US indications for prenatal MR imaging were ventricular dilation $(n=34)$, midline malformations ( $n=$ $20)$, and posterior fossa anomalies $(n=17)$, resulting in agreement with the practice guidelines for the safe and optimal performance of fetal MR imaging of the American College of Radiology and the Society for Pediatric Radiology. ${ }^{1}$

\section{Midline Malformations}

Prenatal MR imaging demonstrated high sensitivity (88.9\%) in detecting midline malformations, with only 3 false-negative findings among 27 cases in this category. The first false-negative finding was callosal hypoplasia in a fetus of 20 weeks, characterized by a clearly thinner-than-normal corpus callosum at postnatal control but with normal length, $>16 \mathrm{~mm}$ in anteroposterior diameter (reference value from our internal normal pool) at prenatal examination. This example of a normally long but abnormally thin corpus callosum may highlight the specific limitations of fetal MR imaging in assessing corpus callosum thickness; abnormalities related to corpus callosum length would be more easily assessable. A previous study has already demonstrated discrepancies between prenatal and postnatal MR imaging related to the corpus callosal 
morphology; however, it investigated a cohort of fetuses with more advanced GA (older than 25 weeks), and it took into account a coarser assessment for the distinction of complete-versuspartial (missing segment) corpus callosum agenesis. ${ }^{10}$ The second false-negative finding was thalamic fusion over the midline in a fetus of 22 weeks. The third false-negative finding was a partial septum pellucidum defect in a fetus of 23 weeks. Despite its minuteness, the septum pellucidum is detectable in $>90 \%$ of fetuses before 27 weeks' GA. ${ }^{15}$ In our case, the incorrect depiction of the septum defect could also be attributed to the less-than-optimal quality of this examination. However, the intrinsic limited spatial resolution of prenatal MR imaging versus postnatal MR imaging, as suggested elsewhere, ${ }^{15}$ is likely to play a pivotal role in all such missed diagnoses.

\section{Disorders of Cortical Development}

In our study, prenatal MR imaging showed a moderate sensitivity $(64.7 \%)$ in detecting disorders of cortical development at an early gestational age. In particular, prenatal MR imaging failed to detect 3 of the 7 polymicrogyrias and 2 of the 3 nodular periventricular heterotopias. Our data are substantially in agreement with those of a previous study by Glenn et al, ${ }^{11}$ which reported sensitivities of $75 \%$ and $44 \%$ in detecting polymicrogyria and heterotopia, respectively, in fetuses younger than 24 weeks' GA. Our experience supports the literature reports that focal cortical anomalies (such as polymicrogyria) appear in their early phase as focal distortion of the cortical plate rim, standing out from an otherwise smooth hemispheric surface, allowing the diagnosis of early developing focal cortical anomalies. ${ }^{16,17}$ The limited sensitivity in detecting heterotopic periventricular nodules is probably due to the their very small size and their signal intensity similar to that of the adjacent germinal matrix remnants. Indeed, when the germinal matrix decreases in thickness with increasing gestational age, the heterotopic nodules may be more easily detected. ${ }^{11}$ However, irregular borders of the lateral ventricles could be retrospectively detected in 1 (25 weeks' GA) of the 2 fetuses with periventricular nodular heterotopias undetected at prenatal MR imaging (On-line Fig 2A, - B). This finding suggests a possible ependymalgerminal layer insult, which evolved into a heterotopia on the postnatal MR imaging from an early triggered cell proliferationmigration deficit.

\section{Posterior Fossa Anomalies}

The false-negative findings among posterior fossa anomalies were 1 molar tooth malformation and 1 Chiari type I malformation. Molar tooth malformation has been reported rarely in prenatal MR imaging literature, basically as single case reports. ${ }^{18-20}$ No sensitivity data are available in this regard: the 2 relatively largest series (of 3 and 7 cases of molar tooth malformation), with a total of 4 cases with early diagnoses (before 25 weeks' GA) by MR imaging or US, were affected by a selection bias; because these cases were within a possible recurrence exclusion prospective protocol, the scanning technique and radiologist were likely to be particularly focused on highlighting minimal heralding signs. ${ }^{21,22}$ At an early GA, besides the presence of a smaller vermis and abnormal fourth ventricle shape on midsagittal sections, the definitive evidence of a molar tooth footprint in the midbrain-superior cerebellar peduncle complex may often be visible in only 1 single axial section, unless more axial acquisitions are acquired with different tilting though the brain stem under the guidance of an a priori hypothesis. In our fetus at 22 weeks' GA, we reported a smaller vermis according to the reference data, ${ }^{23}$ with an anteroposterior diameter of $4 \mathrm{~mm}$ and a superior-inferior diameter of 7 $\mathrm{mm}$, but we did not detect the molar tooth footprint in midbrainsuperior cerebellar peduncles, which was visible on only 1 section (On-line Fig $1 B$ and $-C$ ). The missed prenatal diagnosis of Chiari type I malformation in a fetus undergoing MR imaging for ventricular dilation on US is easy to explain because, to the best of our knowledge, this malformation has not been reported in prenatal imaging and neonatal cases are exceedingly rare. ${ }^{24}$ The progressive herniation of cerebellar tonsils, characteristic of Chiari type I, seems indeed to be the result of a postnatal phenomenon.

Prenatal MR imaging misdiagnosed 3 cases of vermian counterclockwise cranial rotation as pathologic. In such cases, the vermian rotation was isolated and of mild-to-moderate range $\left(<25^{\circ}\right)$, with a normal-sized posterior fossa and normal tentorial insertion and vermian dimensions, thus suggesting a persistent Blake pouch cyst. ${ }^{25}$ Sometimes the Blake pouch cyst disappears by the third trimester due to late fenestration, and the cranial vermian rotation could be detected only until 24-26 weeks. Therefore, isolated mild-to-moderate vermian rotation detected at an early GA does not necessarily indicate an adverse outcome, and in onethird of cases, it undergoes spontaneous resolution in utero, ${ }^{25}$ explaining our false-positive findings. However, even if less common, vermian rotation is reported to be associated with other anomalies in up to $25 \%$ of cases, and in $<10 \%$ of survivors, it is associated with abnormal postnatal neurologic development, ${ }^{26}$ thus giving reason to include it among our prenatal MR imaging findings.

\section{Ventricular/Subarachnoid Space Anomalies}

Our results demonstrated the high accuracy of prenatal MR imaging in depicting the ventricular and subarachnoid space condition due to the high contrast between the signal of the CSF and that of solid adjacent structures. Our results confirm that prenatal MR imaging plays an important role as an adjunctive tool to US in the evaluation of ventricular and subarachnoid spaces because it can rule out pathologic findings at US, confirm the findings, or add associated abnormalities not amenable to US diagnosis. $^{27}$

\section{Vascular Malformations}

Our results about the accuracy of the prenatal MR imaging in detecting vascular malformations are limited by the small incidence of this pathologic finding in general and in our cohort. The only false-negative finding refers to a persistent falcine sinus, not an uncommon incidental finding in the pediatric population. ${ }^{28}$ This diagnostic error may be attributed to the low spatial resolution of the technique used. Furthermore, the falcine sinus normally closes before or shortly after birth, and the detection of this structure in the fetus does not always represent a pathologic finding, except in case of its association with other vascular anomalies such as a vein of Galen malformation or sinus thrombosis. In our case, the persistent falcine sinus was not 
associated with additional vascular anomalies but with a midline malformation (fused thalami) and a cortical gyration disorder (polymicrogyria).

There are many limitations in our study. First, we included only fetuses with postanal MR imaging, usually those with findings suspicious for malformations at prenatal MR imaging, thus resulting in an inflation of the true-positive rate and an underestimation of the true-negative rate. We decided to exclude the cases having undergone pathology for 2 main reasons: 1) The number of cases with pathologic assessment was very limited, and 2) pathology results are often difficult match with fetal MR imaging ones. In this regard, fetal brain pathology may have some paradoxical limitations with respect to fetal MR imaging, especially regarding younger fetal age as in our case. Sometimes, for example, an "agenesis of corpus callosum" is unexpectedly called by postmortem examination, while the corpus callosum had simply collapsed due to postmortem changes and intrapartum brain deformation.

Another limitation is that we relied for the diagnosis on the judgment of pediatric neuroradiologists with long experience in the field, whose learning curve was probably close to a plateau. However, as in other fields of diagnostic imaging, younger professionals should gain their skill working side by side with more experienced professionals before reaching a sufficient plateau of knowledge. Thus, we think that repeating our diagnostic accuracy test with less experienced observers would not match the practice in the main international prenatal MR imaging centers, which generally rely on experienced professionals to whom most cases should be referred, thus avoiding sporadic fetal MR imaging practice in smaller institutions. Furthermore, the readers were from the same institution, and local trends in assessing unclear findings (such as vermian abnormalities) may have influenced the results. We cannot rule out some variability in results if other institutions replicated our work. Finally, currently there are no internationally accepted criteria for referring fetal cases to MR imaging, such as in the case of twin-to-twin transfusion, and they may vary from center to center, so our data base population and our results would not necessarily overlap those of other clinics.

\section{CONCLUSIONS}

The diagnostic value of prenatal MR imaging for brain malformations within 25 weeks of GA is very high, despite important limitations of sensitivity in the early detection of disorders of cortical development, such as polymicrogyrias and periventricular nodular heterotopias.

\section{REFERENCES}

1. ACR-SPR practice guideline for the safe and optimal performance of fetal magnetic resonance imaging (MRI). American College of Radiology (ACR); 2010. 10 p. http://www.guidelines.gov/content. aspx?id $=32509$. Accessed October 1, 2010

2. Sharma G, Heier L, Kalish R, et al. Use of fetal magnetic resonance imaging in patients electing termination of pregnancy by dilation and evacuation. Am J Obstet Gynecol 2003;189:990-93 CrossRef Medline

3. de Laveaucoupet J, Audibert F, Guis F, et al. Fetal magnetic resonance imaging (MRI) of ischemic brain injury. Prenat Diagn 2001; 21:729-36 CrossRef Medline
4. Garel C, Delezoide AL, Elmaleh-Berges M, et al. Contribution of fetal MR imaging in the evaluation of cerebral ischemic lesions. AJNR Am J Neuroradiol 2004;25:1563-68 Medline

5. Tilea B, Delezoide AL, Khung-Savatovski S, et al. Comparison between magnetic resonance imaging and fetopathology in the evaluation of fetal posterior fossa non-cystic abnormalities. Ultrasound Obstet Gynecol 2007;29:651-59 CrossRef Medline

6. Blaicher W, Bernaschek G, Deutinger J, et al. Fetal and early postnatal magnetic resonance imaging: is there a difference? J Perinat Med 2004;32:53-57 Medline

7. Falip C, Blanc N, Maes E, et al. Postnatal clinical and imaging follow-up of infants with prenatal isolated mild ventriculomegaly: a series of 101 cases. Pediatr Radiol 2007;37:981-89 CrossRef Medline

8. Limperopoulos C, Robertson RL Jr, Khwaja OS, et al. How accurately does current fetal imaging identify posterior fossa anomalies? $A J R$ Am J Roentgenol 2008;190:1637-43 CrossRef Medline

9. Senapati G, Levine D, Smith C, et al. Frequency and cause of disagreements in imaging diagnosis in children with ventriculomegaly diagnosed prenatally. Ultrasound Obstet Gynecol 2010;36: 582-95 CrossRef Medline

10. Dhouib A, Blondiaux E, Moutard ML, et al. Correlation between pre- and postnatal cerebral magnetic resonance imaging. Ultrasound Obstet Gynecol 2011;38:170-78 CrossRef Medline

11. Glenn OA, Cuneo AA, Barkovich AJ, et al. Malformations of cortical development: diagnostic accuracy of fetal MR imaging. Radiology 2012;263:843-55 CrossRef Medline

12. Brisse H, Sebag G, Fallet C, et al. Prenatal MRI of corpus callosum agenesis: study of 20 cases with neuropathological correlations [in French]. J Radiol 1998;79:659-66 Medline

13. Tang PH, Bartha AI, Norton ME, et al. Agenesis of the corpus callosum: an MR imaging analysis of associated abnormalities in the fetus. AJNR Am J Neuroradiol 2009;30:257-63 CrossRef Medline

14. Azoulay R, Fallet-Bianco C, Garel C, et al. MRI of the olfactory bulbs and sulci in human fetuses. Pediatr Radiol 2006;36:97-107 CrossRef Medline

15. Schmook MT, Brugger PC, Weber M, et al. Forebrain development in fetal MRI: evaluation of anatomical landmarks before gestational week 27. Neuroradiology 2010;52:495-504 CrossRef Medline

16. Righini A, Zirpoli S, Mrakic F, et al. Early prenatal MR imaging diagnosis of polymicrogyria. AJNR Am J Neuroradiol 2004;25: 343-46 Medline

17. Righini A, Parazzini C, Doneda C, et al. Early formative stage of human focal cortical gyration anomalies: fetal MRI. AJR Am J Roentgenol 2012;198:439-47 CrossRef Medline

18. Fluss J, Blaser S, Chitayat D, et al. Molar tooth sign in fetal brain magnetic resonance imaging leading to the prenatal diagnosis of Joubert syndrome and related disorders. J Child Neurol. 2006;21: 320-24 CrossRef Medline

19. Shen O, Ben-Sira L, Rosenak D, et al. Early second-trimester molar tooth sign. Fetal Diagn Ther 2014;36:259-62 CrossRef Medline

20. Iskender CT, Tarım E, Alkan O. Joubert syndrome and related disorders, prenatal diagnosis with ultrasound and magnetic resonance imaging. J Turk Ger Gynecol Assoc 2012;13:135-38 CrossRef Medline

21. Saleem SN, Zaki MS. Role of MR imaging in prenatal diagnosis of pregnancies at risk for Joubert syndrome and related cerebellar disorders. AJNR Am J Neuroradiol 2010;31:424-29 CrossRef Medline

22. Quarello E, Molho M, Garel C, et al. Prenatal abnormal features of the fourth ventricle in Joubert syndrome and related disorders. $U l$ trasound Obstet Gynecol 2014;43:227-32 CrossRef Medline

23. Parazzini C, Righini A, Rustico M, et al. Prenatal magnetic resonance imaging: brain normal linear biometric values below 24 gestational weeks. Neuroradiology 2008;50:877-83 CrossRef Medline

24. Righini A, Parazzini C, Doneda C, et al. Fetal MRI features related to 
the Chiari malformations. Neurol Sci 2011;32(suppl 3):S279-81 CrossRef Medline

25. Robinson AJ. Inferior vermian hipoplasia: preconception, misconception. Ultrasound Obstet Gynecol 2014;43:123-36 CrossRef Medline

26. Gandolfi Colleoni G, Contro E, Carletti A, et al. Prenatal diagnosis and outcome of fetal posterior fossa fluid collections. Ultrasound Obstet Gynecol 2012;39:625-31 CrossRef Medline
27. Saleem SN. Fetal MRI: an approach to practice-a review. J Adv Res 2014;5:507-23 CrossRef Medline

28. Smith A, Choudhary AK. Prevalence of persistent falcine sinus as an incidental finding in the pediatric population. AJR Am J Roentgenol 2014;203:424-25 CrossRef Medline

29. Garel C. MRI of the Fetal Brain: Normal Development and Cerebral Pathologies. Berlin: Springer-Verlag; 2004 